\title{
Implementações Eficientes da Heurística Min-Min para o HCSP em GPU
}

\author{
Rafael F. Schmid ${ }^{1}$, Edson N. Cáceres ${ }^{1}$ \\ ${ }^{1}$ Faculdade de Computação \\ Universidade Federal de Mato Grosso do Sul \\ Campo Grande, MS - Brasil \\ rafaelfschmidegmail.com, edson@facom.ufms.br
}

\begin{abstract}
Min-min heuristic is one of the most indicated algorithm to solve HCSP, when we are looking for performance. A previous result presented an efficient implementation to this problem with assymptotic complexity $O(t \log (t) m)$. In this work, we implement this problem with linear complexity. Our implementation is competitive with the previous results.
\end{abstract}

\section{Introdução}

A utilização de grades computacionais e computação em nuvem têm obtido bons resultados na solução de problemas complexos. De uma forma geral, grades computacionais e computação em nuvem são compostas de vários comutadores heterogêneos que são capazes de trabalhar de forma cooperativa.

Considerando que temos várias tarefas a serem executadas num conjunto heterogêneo de computadores, onde o tempo de execução de uma tarefa pode variar de acordo com o computador a ser utilizado, temos que resolver o problema de como escalonar as tarefas para serem resolvidas nos computadores no menor tempo possível. Nesses casos, temos uma competição entre as tarefas para utilizar o computador que seja capaz de resolvê-las no menor tempo [Nesmachnow and Canabé 2011]. Esse problema é denominado o Problema de Escalonamento em Ambientes de Computação Heterogênea (HCSP - Heterogeneous Computing Scheduling Problem).

Como trata-se de um problema NP-completo, muitas heurísticas tem sido aplicadas para resolvê-lo, como min-min, max-min, Algoritmos Genéticos (GA), Genetic Simulated Annealing (GSA), Sufferage, etc [Braun et al. 2001]. Em função de suas características, a heurística min-min se mostrou como uma das mais rápidas para esse problema e apresenta soluções quase tão boas quanto GA, que é uma das heurísticas mais demoradas [Wu et al. 2000].

Nesse trabalho, baseado na implementação híbrida de [Pedemonte et al. 2016] e no trabalho de [Schmid et al. 2016], desenvolvemos uma implementação usando a heurística min-min para o HCSP. Nosso resultado tem complexidade linear e a sua implementação se mostrou competitiva em relação aos resultados anteriores.

\section{O problema do HCSP e a Heurística Min-Min}

No modelo apresentado nesse trabalho, todas as tarefas podem ser executadas independentemente, sem se preocupar com a ordem de execução, e quando uma tarefa inicia ela é executada até o final. As aplicações a serem executadas são compostas por uma 
coleção de tarefas indivisíveis, que não tem dependência entre elas, comumente chamadas de metatask. Problemas de escalonamento consistem em tentar minimizar o tempo gasto para executar todas essas tarefas. A formalização abaixo apresenta o modelo matemático para o HCSP com o intuito de minimizar o makespan (tempo gasto a partir do momento em que a primeira tarefa começa até o momento em que a última é completada [Nesmachnow and Canabé 2011]):

- Dado um sistema de computação heterogênea composto de um conjunto de $M$ máquinas $P=m_{1}, m_{2}, \ldots, m_{M}$ e uma coleção de $N$ tarefas $T=t_{1}, t_{2}, . ., t_{N}$ a serem executadas.

- Considere uma função de tempo de execução $E T: P \times T \rightarrow R^{+}$, onde $E T\left(t_{i}, m_{j}\right)$ é o tempo necessário para executar a tarefa $t_{i}$ na máquina $m_{j}$.

- O objetivo do HCSP é encontrar uma atribuição de tarefas às máquinas (uma função $f: T^{N} \rightarrow P^{M}$ ) que minimize o makespan, conforme definido na Equação 1.

$$
\max _{m_{j} \in P} \sum_{\substack{t_{i} \in T \\ f\left(t_{i}\right)=m_{j}}} E T\left(t_{i}, m_{j}\right)
$$

A heurística min-min começa com o conjunto $U$ de todas as tarefas não mapeadas. Então, o conjunto de máquinas de menor tempo de conclusão $M$ para cada tarefa em $U$ é encontrado. A tarefa cujo tempo de conclusão seja o menor, é escolhida e atribuída à máquina correspondente. A nova tarefa mapeada é removida do conjunto $U$, e o processo se repete até que todas as tarefas sejam mapeadas [Braun et al. 2001].

\section{Trabalhos Relacionados}

Nesmachnow e Canabé (2011) apresentaram uma implementação em GPU para a heurística min-min e obtiveram speedups de aproximadamente seis vezes em relação a uma implementação sequencial. Ezzatti et al. (2013) propuseram um algoritmo min-min, que chamamos de min-min sort, que efetua a ordenação segmentada sobre os tempos que cada máquina leva para executar cada uma das tarefas, e depois verifica, nessa ordem, as tarefas de cada máquina, selecionando a menor para aquela iteração. Se utilizado um algoritmo de ordenação linear, esse algoritmo alcança complexidade assintótica também linear: $O(t m)$, onde $t$ é o número de tarefas e $m$ o número de máquinas, mas quando da implementação os autores utilizaram um algoritmo $O(t \log (t) m)$. Posteriormente, Pedemonte et al. (2016) apresentaram uma implementação híbrida GPU-CPU do min-min sort com resultados melhores que os anteriores.

\section{Solução Proposta e Resultados Experimentais}

Os experimentos deste artigo foram realizados em uma máquina com o Sistema Operacional Ubuntu 16.04, 16 GB de memória RAM, processador Intel Core ${ }^{t m}$ i5-4460 CPU @ 3.20 GHz x 4 e placa gráfica GeForce GTX 1050 Ti com 4 GB de memória, e os cenários de teste utilizados foram obtidos do endereço https: / / par-cga-sched.gforge. uni.lu/instances/etc/.

Baseado no trabalho de [Schmid et al. 2016], apresentamos uma implementação da heurística min-min sort utilizando o radix-sort. O principal objetivo é utilizar uma 
ordenação segmentada que garanta complexidade linear para a heurística min-min. Para efetuar as comparações também foi implementada: a min-min padrão em CPU e GPU; e a min-min sort em CPU, GPU e Híbrida (GPU-CPU).

A implementação em GPU da heurística min-min padrão foi testada utilizando duas estruturas diferentes. A primeira utiliza as linhas da matriz para representar as máquinas e as colunas para representar as tarefas. Cada thread CUDA é responsável por processar uma tarefa, ou seja, encontrar a máquina em que a tarefa possui o menor tempo de conclusão. Cada thread armazena seu resultado em uma posição do vetor resultante, onde é realizada uma redução paralela para encontrar a tarefa que deve ser escalonada naquela iteração. Dessa forma, as threads acessam a mesma linha da matriz a cada iteração (Figura 4), favorecendo a coalescência. Ao inverter essa estrutura perdemos a coalescência e os tempos sobem bastante, por isso optamos não abordá-la aqui.

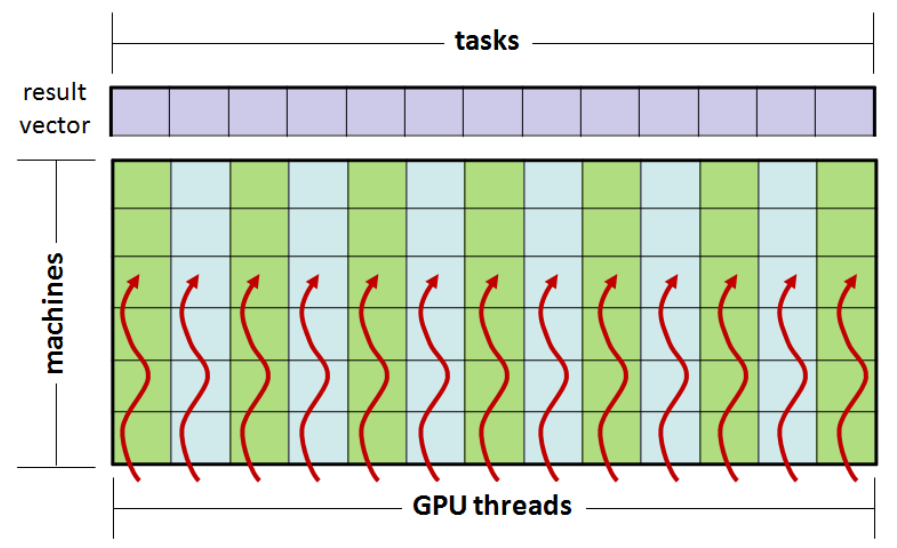

\section{Figura 1. Estratégia de implementação em GPU da heurística min- min [Nesmachnow and Canabé 2011].}

A implementação paralela do min-min sort de [Ezzatti et al. 2013], efetua a ordenação segmentada utilizando uma biblioteca CUDA, e depois utiliza uma redução paralela para identificar as máquinas que possuem o menor tempo de conclusão na iteração corrente. Para essa estratégia representamos as máquinas em linhas e as tarefas em colunas, estrutura necessária para efetuarmos a ordenação segmentada de forma eficiente.

A nossa implementação do min-min sort híbrido CPU-GPU usa a mesma estrutura da versão paralela do algoritmo min-min sort. Na primeira etapa, a ordenação das linhas da matriz, é efetuada em GPU. Posteriormente, os dados são copiados de volta para CPU e a mesma implementação da versão sequencial do min-min sort é executada. Para a ordenação utilizamos a biblioteca CUB (radix-sort).

A Figura 2 apresenta o speedup de cada uma das implementações: baseadas no merge-sort (Pedemont et al.) e baseadas no radix-sort (implementação proposta) quando comparadas com a implementação sequencial do min-min padrão. Com isso, podemos observar que, apesar de estarmos utilizando um algoritmo linear, em termos de desempenho, o comportamento é semelhante ao algoritmo de complexidade $O(t \log (t) m)$ da biblioteca MGPU.

Podemos notar também, que a implementação sequencial do min-min sort apresenta melhores resultados para matrizes menores, já que o acesso não é coalescido, devido 
a ordenação segmentada do início do algoritmo. O motivo de apresentar melhores resultados é que a ordenação segmentada em GPU possui desempenho superior à ordenação sequencial, prova disso são os desempenhos bem melhores das implementações híbridas.

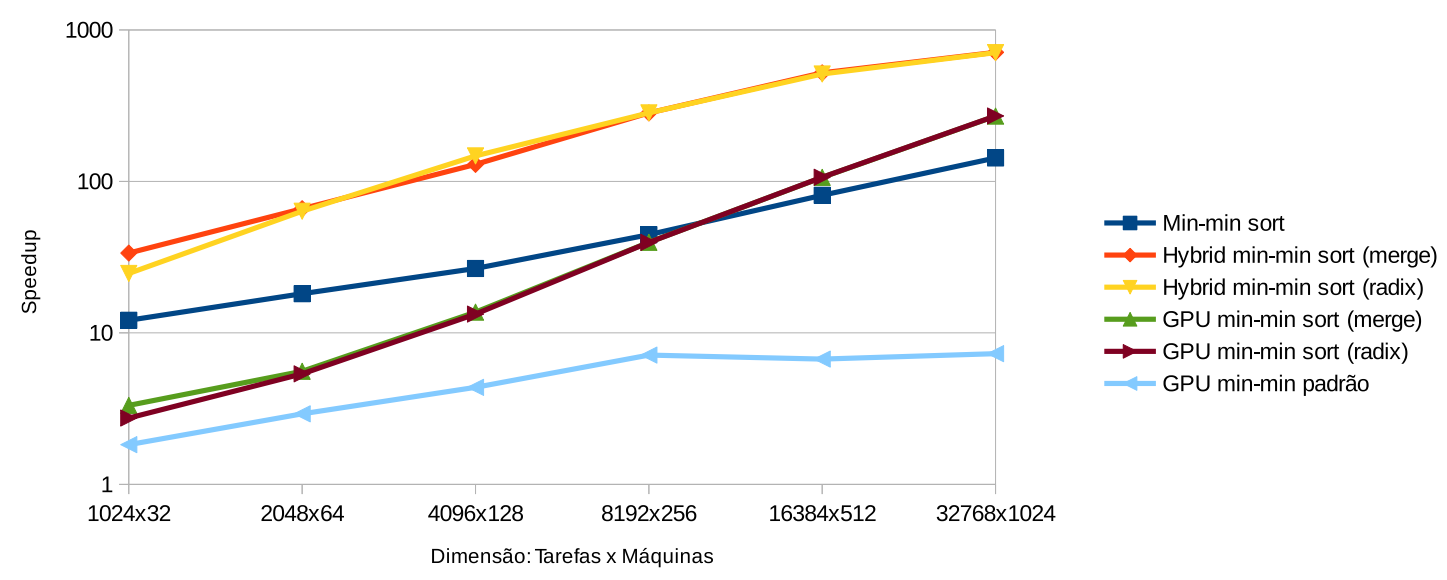

Figura 2. Speedup das implementações do Min-Min.

\section{Conclusões}

A implementação que apresentamos possui complexidade linear e os resultados obtidos na GPU e GPU-CPU são competitivos.

\section{Referências}

Braun, T. D., Siegel, H. J., Beck, N., Bölöni, L. L., Maheswaran, M., Reuther, A. I., Robertson, J. P., Theys, M. D., Yao, B., Hensgen, D., and Freund, R. F. (2001). A comparison of eleven static heuristics for mapping a class of independent tasks onto heterogeneous distributed computing systems. Journal of Parallel and Distributed Computing, 61(6):810 - 837.

Ezzatti, P., Pedemonte, M., and Martín, Á. (2013). An efficient implementation of the min-min heuristic. Computers \& Operations Research, 40(11):2670-2676.

Nesmachnow, S. and Canabé, M. (2011). Gpu implementations of scheduling heuristics for heterogeneous computing environments. In XVII Congreso Argentino de Ciencias de la Computación.

Pedemonte, M., Ezzatti, P., and Martín, Á. (2016). Accelerating the min-min heuristic. In Parallel Processing and Applied Mathematics, pages 101-110. Springer.

Schmid, R., Pisani, F., Borin, E., and Cáceres, E. (2016). An evaluation of segmented sorting strategies on gpus. In IEEE 18th International Conference on High Performance Computing and Communications (HPCC), pages 1123-1130. IEEE.

Wu, M.-Y., Shu, W., and Zhang, H. (2000). Segmented min-min: A static mapping algorithm for meta-tasks on heterogeneous computing systems. In Heterogeneous Computing Workshop, 2000.(HCW 2000) Proceedings. 9th, pages 375-385. IEEE. 